\title{
Roux-en-y gastric bypass attenuates hepatic mitochondrial dysfunction in mice with non-alcoholic steatohepatitis
}

\author{
Jef Verbeek, ${ }^{1}$ Matthias Lannoo, ${ }^{2}$ Eija Pirinen, ${ }^{3,4}$ Dongryeol Ryu, ${ }^{3}$ Pieter Spincemaille, ${ }^{5}$ \\ Ingrid Vander Elst, ${ }^{1}$ Petra Windmolders, ${ }^{1}$ Karin Thevissen, ${ }^{5}$ Bruno P A Cammue, ${ }^{5,6}$ \\ Jos van Pelt, ${ }^{1}$ Sabine Fransis, ${ }^{7}$ Peter Van Eyken, ${ }^{7}$ Chantal Ceuterick-De Groote, ${ }^{8}$ \\ Paul P Van Veldhoven, ${ }^{9}$ Pierre Bedossa, ${ }^{10}$ Frederik Nevens, ${ }^{1}$ Johan Auwerx ${ }^{3}$ \\ David Cassiman ${ }^{1,11}$
}

- Additional material is published online only. To view please visit the journal online (http://dx.doi.org/10.1136/ gutjnl-2014-306748).

For numbered affiliations see end of article.

\section{Correspondence to} Dr Jef Verbeek, Department of Hepatology, University Hospitals KU Leuven, Herestraat 49, Leuven 3000, Belgium;

jef.verbeek@uzleuven.be

Received 9 January 2014 Revised 30 April 2014 Accepted 23 May 2014

Published Online First

10 June 2014

\section{SLinked}

http://dx.doi.org/10.1136/ gutjnl-2014-307738

\section{CrossMark}

To cite: Verbeek Lannoo M, Pirinen $\mathrm{E}$, et al. Gut 2015:64:673-683.

\section{ABSTRACT}

Objective No therapy for non-alcoholic steatohepatitis (NASH) has been approved so far. Roux-en-y gastric bypass (RYGB) is emerging as a therapeutic option, although its effect on NASH and related hepatic molecular pathways is unclear from human studies. We studied the effect of RYGB on pre-existent NASH and hepatic mitochondrial dysfunction - a key player in NASH pathogenesis - in a novel diet-induced mouse model nicely mimicking human disease.

Design (57BL/6J mice were fed a high-fat highsucrose diet (HF-HSD).

Results HF-HSD led to early obesity, insulin resistance and hypercholesterolaemia. HF-HSD consistently induced NASH (steatosis, hepatocyte ballooning and inflammation) with fibrosis already after 12-week feeding. NASH was accompanied by hepatic mitochondrial dysfunction, characterised by decreased mitochondrial respiratory chain (MRC) complex I and IV activity, ATP depletion, ultrastructural abnormalities, together with higher 4-hydroxynonenal (HNE) levels, increased uncoupling protein 2 (UCP2) and tumour necrosis factor- $\alpha$ (TNF- $\alpha$ ) mRNA and free cholesterol accumulation. In our model of NASH and acquired mitochondrial dysfunction, RYGB induced sustained weight loss, improved insulin resistance and inhibited progression of NASH, with a marked reversal of fibrosis. In parallel, RYGB preserved hepatic MRC complex I activity, restored ATP levels, limited HNE production and decreased TNF- $\alpha$ mRNA.

Conclusions Progression of NASH and NASH-related hepatic mitochondrial dysfunction can be prevented by RYGB. RYGB preserves respiratory chain complex activity, thereby restoring energy output, probably by limiting the amount of oxidative stress and TNF- $\alpha$. These data suggest that modulation of hepatic mitochondrial function contributes to the favourable effect of RYBG on established NASH.

\section{INTRODUCTION}

Non-alcoholic fatty liver disease (NAFLD) has become one of the most prevalent liver diseases in our sedentary Western society. ${ }^{1}$ Its prevalence is rising in parallel with that of the metabolic syndrome and now already $20-30 \%$ of adults

\section{Significance of this study}

What is already known on this subject?

- Non-alcoholic steatohepatitis (NASH) will become the most common indication for liver transplantation. No efficient therapy for NASH has been found yet.

- The majority of current NASH mouse models do not fully mimic the clinical reality.

- Mitochondrial dysfunction is associated with fatty liver disease, although data about mitochondrial dysfunction in a representative NASH context are underreported and equivocal.

- Roux-en-y gastric bypass (RYGB) is emerging as a valuable treatment option in obese NASH patients, but despite numerous human studies its impact on histological NASH features is still under debate and remarkably, hepatic molecular effects are unknown.

What are the new findings?

- A high-fat high-sucrose diet fed to mice is able to induce NASH with fibrosis after only 12 weeks, together with early obesity, insulin resistance and hypercholesterolaemia.

- Detailed hepatic profiling revealed decreased mitochondrial respiratory chain complex activities, ATP depletion and ultrastructural mitochondrial abnormalities in conjunction with $\mathrm{NASH}$, probably caused by increased respiratory uncoupling, oxidative stress, increased tumour necrosis factor- $\alpha$ (TNF- $\alpha$ ) and free cholesterol accumulation.

- RYGB halts the progression of pre-existing NASH and reverses fibrosis in our model.

- RYGB attenuates hepatic mitochondrial dysfunction (preserving respiratory complex activity and restoring ATP levels), probably by limiting the amount of oxidative stress and TNF- $\alpha$.

are affected. ${ }^{2}$ NAFLD comprises a disease spectrum ranging from isolated steatosis to non-alcoholic steatohepatitis (NASH), which is hallmarked by 
Significance of this study

\section{How might it impact on clinical practice in the foreseeable future?}

- These data suggest that modulation of mitochondrial function contributes to the beneficial effect of RYGB on NASH and thereby provide further insight in the mode of action of the most frequently applied bariatric procedure in patients.

- Our work underscores the position of RYGB as a therapeutic modality in NASH.

- This improved NASH mouse model to study pathophysiology and test therapeutic options should facilitate translation from the mouse, back to humans.

steatosis in combination with hepatocyte ballooning and inflammation with or without fibrosis. ${ }^{3}$ NASH can progress to endstage liver disease and related complications, including hepatocellular carcinoma. ${ }^{4}$ At present, NASH is the third indication for liver transplantation in the USA and is on its way to becoming the main indication between 2020 and 2025. ${ }^{5}$ Furthermore, NASH has been identified as an independent risk factor for cardiovascular diseases. ${ }^{6}$

The societal and medical burden of NAFLD boosted a large amount of studies on pathophysiology and treatment of NASH. However, till now these research efforts have not led to a magic therapeutic bullet. Dietary modifications and physical activity remain the cornerstone of treatment, although with rather disappointing efficacy in patients we encounter in daily practice. ${ }^{7}$ Due to inconsistent results in rodent studies and clinical trials, no pharmacological agent has been approved for the treatment of NASH yet. ${ }^{89}$

As a consequence, bariatric surgery has emerged as a possibly more effective therapy for NASH in obese patients. ${ }^{10}{ }^{11}$ Roux-en-y gastric bypass (RYGB), the most common bariatric procedure, is considered as a potent therapy for obesity and insulin resistance, both major players in the development of NASH. ${ }^{12}$ Studies consistently show a decrease in liver steatosis after RYGB; however, the evolution in hepatocyte ballooning, inflammation and fibrosis remains subject to debate. ${ }^{11}{ }_{13}$ Moreover, the effect of RYGB on hepatic molecular pathways involved in NASH pathophysiology remains a highly underexplored research area that is hampered by the difficulty of obtaining human liver tissue once bariatric surgery has been performed. Therefore, a mouse model that displays NASH and wherein subsequently RYGB is carried out could serve as a valuable surrogate research tool. Unfortunately, the majority of current rodent NASH models do not fully mimic the clinical reality with respect to histological pattern or metabolic profile. $^{1415}$

Hepatic mitochondrial dysfunction is thought to participate at various levels in NASH development and therefore could represent an attractive molecular target that mediates the effects of RYGB. But literature reveals many discrepancies regarding mitochondrial respiratory chain (MRC) complex activity, oxidative phosphorylation efficiency, ATP levels and mitochondrial DNA (mtDNA) content in NAFLD, as recently reviewed. ${ }^{16}{ }^{17}$ The discrepancies can be explained by differences in study design with respect to the stage of NAFLD (isolated steatosis to moderate or severe NASH), the age and type of research object (mice, rats, humans) and the method of induction of experimental NAFLD. Altogether, studies on mitochondrial function in a representative NASH model are largely underreported and equivocal.

To address these issues, we used a high-fat high-sucrose diet (HF-HSD)-induced mouse model of NASH and fibrosis, with close resemblance to clinical practice and that is convenient to use for research purposes. Mitochondrial function and its potential mediators were profiled in detail. Ultimately, we performed RYGB in these NASH mice and explored its capacities to combat NASH and modulate mitochondrial function.

\section{MATERIALS AND METHODS \\ Animals and diet}

Male C57BL/6J mice (The Jackson Laboratory, Bar Harbor, Maine, USA) were housed under a $14 \mathrm{~h}$ light $-10 \mathrm{~h}$ dark cycle at $21-23^{\circ} \mathrm{C}$ and had ad libitum access to water during the entire experiment. From the age of 6 weeks, mice were fed a 'Western' HF-HSD with $44.6 \%$ of kcal derived from fat (of which $61 \%$ saturated fatty acids) and $40.6 \%$ of kcal derived from carbohydrates (primarily sucrose $340 \mathrm{~g} / \mathrm{kg}$ diet) (TD.08811, 45\% kcal Fat Diet, Harlan Laboratories Inc., Madison, Wisconsin, USA) or normal chow diet (NCD) as control (V1534-000 ssniff R/M-H, ssniff Spezialdiäten GmbH, Soest, Germany) for 4, 12 and 20 weeks ( $n=8$ per group for every time point), where after they were sacrificed. All procedures were approved by the animal welfare committee of the University of Leuven.

\section{In vivo phenotyping}

Body weight and food intake were monitored weekly on the same day. After sedation with sodium pentobarbital (intraperitoneal injection, $50 \mathrm{mg} / \mathrm{kg}$ body weight), total fat mass was analysed by dual-energy X-ray absorptiometry (DEXA) (PIXImus densitometer, Lunar Corp., Madison, Wisconsin, USA). Intraperitoneal glucose tolerance test (IPGTT) was performed in $6 \mathrm{~h}$ fasted mice. Tail vein glucose levels were measured with a Bayer Contour glucometer immediately before (time point $0 \mathrm{~min}$ ) and 15, 30, 60, 90 and $150 \mathrm{~min}$ after glucose administration (1 $\mathrm{g}$ glucose $/ \mathrm{kg}$ bodyweight). Insulin resistance was calculated using the Homeostasis Model of Insulin Resistance (HOMA-IR) index: (fasting insulin $(\mathrm{ng} / \mathrm{mL}) \times$ fasting glucose $(\mathrm{mg} / \mathrm{dL})) / 405 .{ }^{18}$

\section{Sacrifice}

After a $6 \mathrm{~h}$ fasting period, mice were anaesthetised with sodium pentobarbital (intraperitoneal injection, $50 \mathrm{mg} / \mathrm{kg}$ body weight) and killed by blood sampling via cardiac puncture. Plasma was obtained by centrifugation of blood $(6000 \mathrm{rpm}$ for $5 \mathrm{~min}$ at $\left.4^{\circ} \mathrm{C}\right)$ that was collected in heparinised syringes. Tissues were either snap frozen in liquid nitrogen and together with the plasma stored at $-80^{\circ} \mathrm{C}$ till further biochemical and molecular analyses or preserved for histological analysis.

\section{Histological analyses}

Liver samples were routinely fixed in buffered formalin (4\%) and embedded in paraffin. Serial $4 \mu \mathrm{m}$ thick sections were stained with $\mathrm{H} \& \mathrm{E}$ and picrosirius red to assess fibrosis. Frozen liver sections were stained with Oil Red O to assess lipid accumulation. All liver biopsies were analysed by an expert liver pathologist, blinded to the dietary condition or surgical intervention. Steatosis, activity and fibrosis were semiquantitatively scored according to the NASH-Clinical Research Network criteria. ${ }^{19}$ The amount of steatosis (percentage of hepatocytes containing fat droplets) was scored as $0(<5 \%), 1(5-33 \%)$, $2(>33-66 \%)$ and 3 (>66\%). Hepatocyte ballooning was 
classified as 0 (none), 1 (few) or 2 (many cells/prominent ballooning). Foci of lobular inflammation were scored as 0 (no foci), 1 ( $<2$ foci per $200 \times$ field), 2 ( $2-4$ foci per $200 \times$ field) and 3 ( $>4$ foci per $200 \times$ field). Fibrosis was scored as stage F0 (no fibrosis), stage F1a (mild, zone 3, perisinusoidal fibrosis), stage F1b (moderate, zone 3, perisinusoidal fibrosis), stage F1c (portal/periportal fibrosis), stage F2 (perisinusoidal and portal/ periportal fibrosis), stage F3 (bridging fibrosis) and stage F4 (cirrhosis). Diagnosis of NASH was based on accepted histological criteria. $^{2021}$ Severity of the disease was assessed using the NAS (NAFLD activity score) as the unweighted sum of scores of steatosis, hepatocyte ballooning and lobular inflammation. ${ }^{19}$ Percentage of fibrosis was quantitated by morphometry from digitalised sirius red stained sections using the Aperio system after tuning the threshold of fibrosis detection under visual control. Result is expressed as collagen proportional area. ${ }^{22}$

\section{Mitochondrial assays}

Measurement of complex I activity (Complex I Enzyme Activity Microplate Assay Kit, ab109721, Abcam Mitosciences, Eugene, Oregon, USA) and complex IV activity (Complex IV Rodent Enzyme Activity Microplate Assay Kit, ab109911, Abcam Mitosciences, Eugene, Oregon, USA) of the MRC were performed spectrophotometrically in liver cell lysates according to the manufacturer's instructions. Citrate synthase activity was measured in parallel with the complex I and IV assays (using same liver lysate on same day), following the protocol of Sigma's Citrate Synthase Assay Kit (Sigma-Aldrich, St Louis, Missouri, USA).

To measure mtDNA content (marker of mitochondrial number), liver tissue was homogenised using $1 \times$ phosphate buffered saline (PBS) and digested in a lysis buffer containing Proteinase K overnight. Genomic and mitochondrial DNA were extracted by conventional phenol-chloroform method. Then quantitative PCR was performed using mitochondrial DNA (16S) and genomic DNA (mean of uncoupling protein 2 (UCP2) and hexokinase-II (HK-II)) specific primers as previously described. $^{23}$ ATP content was measured in liver tissue after homogenisation in radio-immunoprecipitation assay (RIPA) lysis buffer $(1 \times$ PBS, 1\% Nonidet P-40, 0.5\% sodium deoxycholate, $0.1 \%$ sodium dodecyl sulphate, $1 \mathrm{mM}$ sodium orthovanadate $\left(\mathrm{Na}_{3} \mathrm{VO}_{4}\right), 200 \mathrm{mM}$ phenylmethanesulfonylfluoride, protease inhibitor cocktail), using the CellTiter-Glo Luminescent Cell Viability Assay (Promega Corp., Madison, Wisconsin, USA) according manufacturer's instructions. 4-Hydroxynonenal (HNE), a by-product of lipid peroxidation and thus a marker of oxidative stress, was measured following manufacturer's protocol (OxiSelect HNE-His Adduct ELISA Kit, Cell Biolabs Inc., San Diego, California, USA). Mitochondrial ultrastructural analysis was performed using standard transmission electron microscopy on glutaraldehyde-fixed liver tissue.

\section{Gastric bypass procedure}

RYGB $(n=12)$ or a SHAM procedure $(n=6)$ was performed in male C57BL/6J mice that in advance were fed HF-HSD for 12 weeks (diet started at age of 6 weeks). After $1 \mathrm{~h}$ fasting, mice were anaesthetised with isoflurane $\left(2-3 \%\right.$ with $\left.\mathrm{O}_{2}\right)$. The mice were placed on a surgical heating pad and preoperative analgesia was administered (carprofen, subcutaneous injection, $5 \mathrm{mg} / \mathrm{kg}$ bodyweight). After shaving and disinfecting the abdominal region, a $4 \mathrm{~cm}$ midline laparotomy was made.

In the RYGB procedure (operating time $\pm 60 \mathrm{~min}$ ), the stomach was divided $3 \mathrm{~mm}$ distally from the gastro-oesophageal junction to create a small gastric pouch. The remnant stomach was closed with an 8-0 Ethilon Nylon Suture. To create the alimentary limb, the jejunum was opened $2 \mathrm{~cm}$ distally from the ligament of Treitz and connected to the small gastric pouch in a side (jejunum)-to-end fashion (9-0 Ethilon Nylon Suture). Just proximal from this anastomosis, the biliary limb was transected with closure of both ends with a purse string suture. Subsequently, $6 \mathrm{~cm}$ (measured in a non-stretched way) distally from the gastrojejunal anastomosis, a new side-to-side anastomosis was made between the alimentary $(6 \mathrm{~cm})$ and the biliopancreatic limb $(4 \mathrm{~cm})$ to create the common channel (not measured) (9-0 Ethilon Nylon Suture). The SHAM procedure (operating time $\pm 20 \mathrm{~min}$ ) consisted of a $5 \mathrm{~mm}$ gastrostomy in the anterior wall (corpus) and closure with an 8-0 Ethilon Nylon Suture. In each mouse, the abdominal wall and skin was closed with a 4-0 coated Vicryl Rapide (polyglactin 910) (continuous suturing). Before closure of the abdomen, buprenorphine (intraperitoneal injection, $0.1 \mathrm{mg} / \mathrm{kg}$ bodyweight) was administered, together with $200 \mu \mathrm{L}$ of $\mathrm{NaCl} 0.9 \%$ to compensate for intraoperative fluid loss.

After surgery, mice were housed individually for the remainder of the experiment. To prevent postoperative pain, all mice received carprofen and buprenorphine (in aforementioned doses) once daily the first 2 days after surgery. HF-HSD was continued in both groups for 8 weeks till sacrifice. Only the first two postoperative days, in both groups we additionally provided manually crushed HF-HSD together with Hydrogel (ClearH20, Portland, Maine, USA) at the bottom of the cage in order to stimulate postoperative recovery. Postoperative in vivo phenotying, sacrifice and subsequent histological, biochemical and molecular analyses were performed as aforementioned. IPGTT was done in week 6 after surgery.

\section{Statistical analyses}

Statistical analyses were performed with GraphPad Prism V.6 (GraphPad Software, La Jolla, California, USA). To determine significant differences between two groups, Student's t (parametric samples) and Mann-Whitney test (non-parametric samples) were used. Significant differences between more than two groups were estimated by one-way analysis of variance and Tukey post hoc analysis (parametric samples) or Kruskal-Wallis and Dunn's multiple comparisons test (non-parametric samples). A p value of $<0.05$ was considered as statistically significant. Significance is represented by ${ }^{*} \mathrm{p}<0.05, * \mathrm{p}<0.01$ and $* * * \mathrm{p}<0.001$. Error bars are SEM.

\section{RESULTS}

\section{HF-HSD induces early obesity, insulin resistance} and hypercholesterolaemia

Compared with mice on NCD, HF-HSD mice exhibited early obesity reflected in a significantly higher body weight from 2 weeks after start of diet (figure 1A), together with increased fat mass measured by DEXA (figure 1B) and increased epididymal fat pad weight (figure 1C), determined after 4, 12 and 20 weeks of diet. Glucose intolerance assessed by IPGTT was present after 4 weeks of HF-HSD feeding and clearly aggravated with longer feeding periods (see online supplementary figure S1A for glucose curves per time point, figure 1D for associated area under the curve values). HOMA-IR index (marker of insulin resistance) determined after 4-, 12- and 20-week diet was significantly higher in HF-HSD mice compared with NCD mice (figure 1E). Plasma total cholesterol tended to be higher after 4-week HF-HSD, reaching significance after 12- and 20 -week diet (figure 1F). We observed no differences in plasma triglycerides (see online supplementary figure S1B). 
A
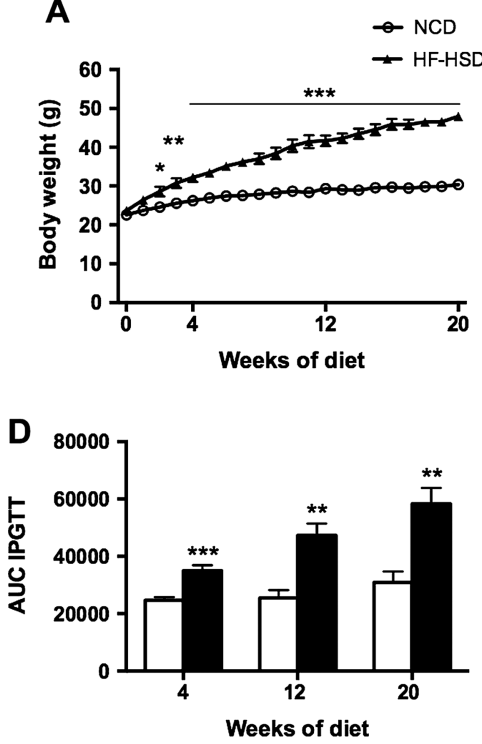

B

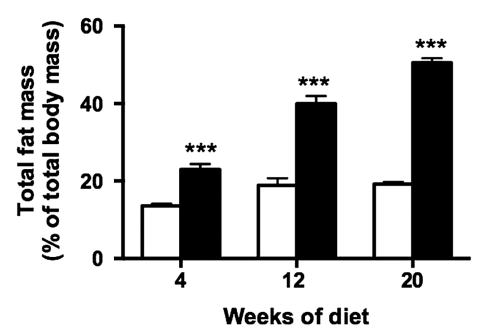

E

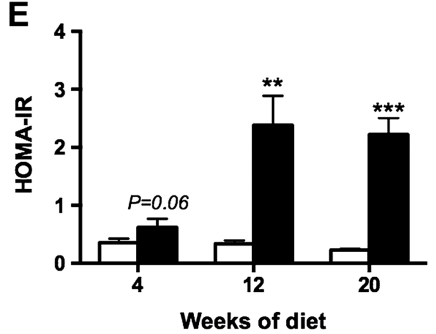

C
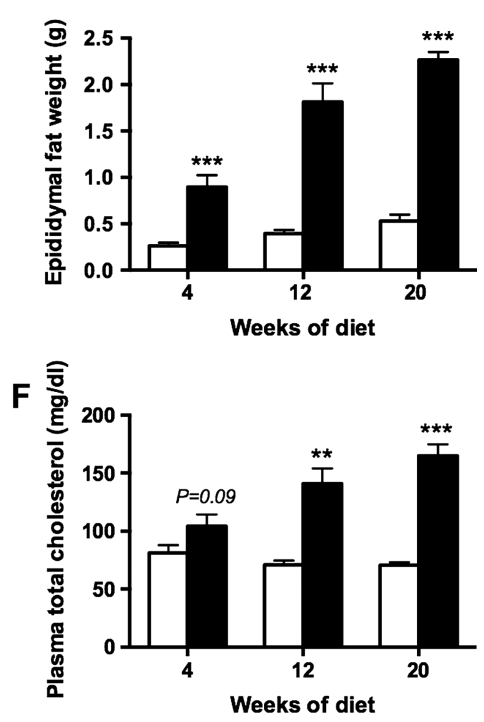

Figure 1 Systemic metabolic effects of high-fat high-sucrose diet (HF-HSD). (A) Progression body weight ( $n=8$ per group). (B) Total fat mass measured by dual-energy X-ray absorptiometry scan ( $n=4$ per group per time point). (C) Epididymal fat pad weight ( $n=8$ per group per time point). (D) Area under the curve of intraperitoneal glucose tolerance test (6 h fasting, $1 \mathrm{~g} / \mathrm{kg}$ glucose) after 4 ( $\mathrm{n}=6$ per group), 12 ( $\mathrm{n}=4 \mathrm{per}$ group) and 20 ( $\mathrm{n}=6$ per group) weeks diet, respectively. (E) Homeostasis Model of Insulin Resistance (HOMA-IR) (fasting glucose (mg/dL) $\times \mathrm{fasting}$ insulin ( $\mathrm{ng} / \mathrm{mL}$ )/ 405) at sacrifice ( $n=6$ per group after 4 weeks and $n=8$ per group after 12- and 20-week diet). (F) Fasting plasma total cholesterol ( $n=8$ per group per time point). Asterisks indicate significant difference versus control group at same time point. Data are presented as mean. Error bars are SEM. White bars represent normal chow diet (NCD) mice; black bars represent HF-HSD mice.

\section{HF-HSD administration for 12 weeks leads to NASH with fibrosis}

Mice on NCD did not show any histological abnormalities after 4-, 12- and 20-week diet. Also, 4-week HF-HSD did not result in significant histological abnormalities. However, 12-week HF-HSD consistently (8/8 mice) led to mainly mediovesicular steatosis (localised centrilobular or without clear zonal distribution) in combination with hepatocyte ballooning and lobular inflammation, compatible with the diagnosis of NASH (figure 2A). Twenty-week HF-HSD feeding even led to more severe NASH, in particular further increase of steatosis. NASH was in the majority of mice accompanied by mainly zone 3 perisinusoidal fibrosis and occasionally periportal fibrosis (see online supplementary figure S2). In correspondence, morphometric assessment showed a significant increase of collagen proportional area in HF-HSD mice compared with NCD mice after 12 weeks of diet, which became more pronounced after 20 weeks (figure 2B). In addition, mRNA levels of genes involved in fibrogenesis were significantly increased after 12and 20-week HF-HSD (figure 2C). Mean histology scores for each dietary condition per time point can be found in online supplementary table S1. In agreement with these histological findings, liver weight (figure 2D) and hepatic triglyceride levels (figure 2E) were higher in HF-HSD versus NCD mice after 12and 20-week diet. Plasma alanine transaminase levels (as a marker of liver damage) were significantly increased in HF-HSD mice from 12-week diet onwards (figure 2F). mRNA levels of genes involved in fatty acid uptake, lipogenesis and endoplasmic reticulum stress can be found in online supplementary figure S3.

\section{Mitochondrial dysfunction in association with NASH in HF-HSD mice}

Absolute MRC complex I and MRC complex IV activities were lower in mice fed HF-HSD for 12 and 20 weeks (time points on which NASH was observed) (figure $3 \mathrm{~A}, \mathrm{~B}$ ). This despite an accompanying increase in citrate synthase activity and, as suggested by gene expression analysis, an increased fatty acid $\beta$-oxidation in HF-HSD mice (figure $3 \mathrm{C}, \mathrm{D}$ ). MRC complex activity was not significantly different between mice that received 4-week NCD and HF-HSD feeding (figure 3A,B). MtDNA levels (as a marker of mitochondrial number) were not different between both dietary conditions at any time point (figure 3E). We observed significant ATP depletion after $12-$ and 20-week HF-HSD feeding (figure $3 \mathrm{~F}$ ). Ultrastructural analysis of mitochondria in hepatic cells showed focal damages of the mitochondrial outer and inner membranes particularly in swollen mitochondria (figure $3 \mathrm{Gc}$ ), together with rarefaction of the matrix and rearranged cristae (figure $3 \mathrm{Gd}$ ) in NASH mice compared with mitochondria of similarly processed control samples of NCD mice (figure $3 \mathrm{Ga}$ and $\mathrm{b}$ ).

Additionally, we assessed potential mediators of the decreased MRC complex activity and energy output in our NASH mice. Several MRC complexes are sensitive to oxidative stress. $^{16} 24$ We observed significantly higher HNE levels (marker of oxidative stress) in HF-HSD mice compared with NCD mice after 20-week feeding (figure 4A). Free cholesterol is increasingly recognised as an important determinant of $\mathrm{NASH}$ pathophysiology and can trigger mitochondrial injury. ${ }^{16} 25$ Interestingly, hepatic free cholesterol levels were higher in HF-HSD mice after 12- and 20-week diet compared with NCD mice (figure 4B). After 20-week HF-HSD, we observed in addition increased TNF- $\alpha$ mRNA, theoretically further impairing MRC complex activity (figure 4C). ${ }^{16}$ UCP2 mRNA was significantly higher in both 12- and 20-week HF-HSD mice, suggesting an increased uncoupling of oxidation from ATP synthesis (figure 4D). We observed no significant differences in saturated fatty acid content (see online supplementary figure S4). 
A
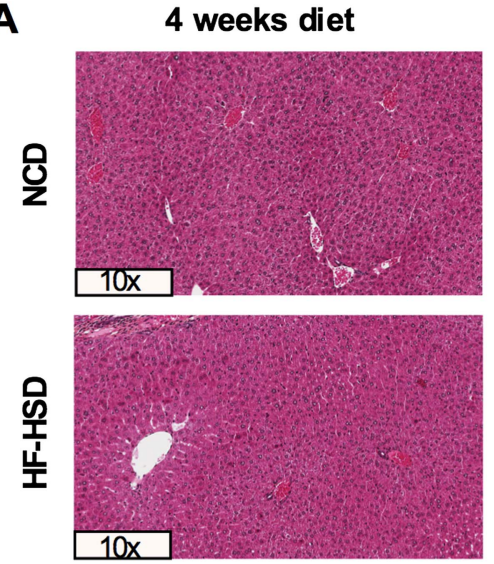

B
12 weeks diet
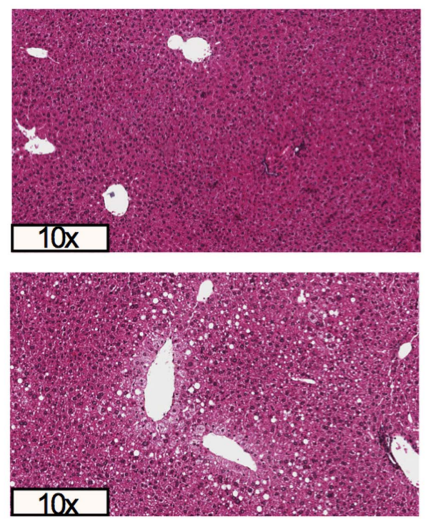

20 weeks diet
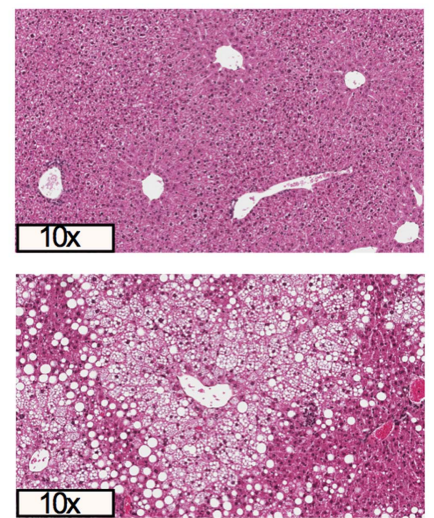

C

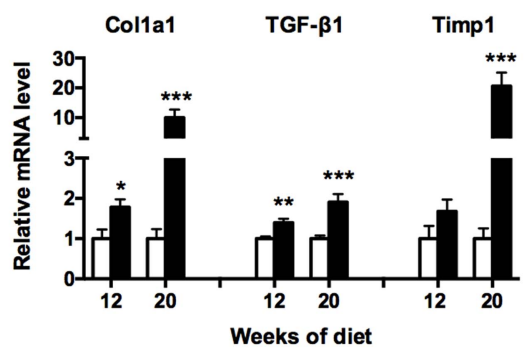

$\mathbf{F}$

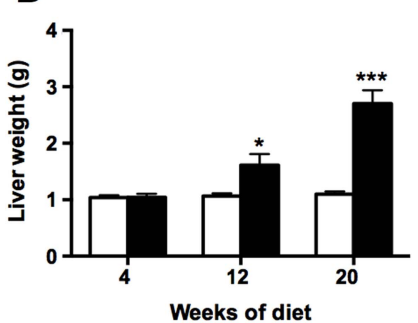

E
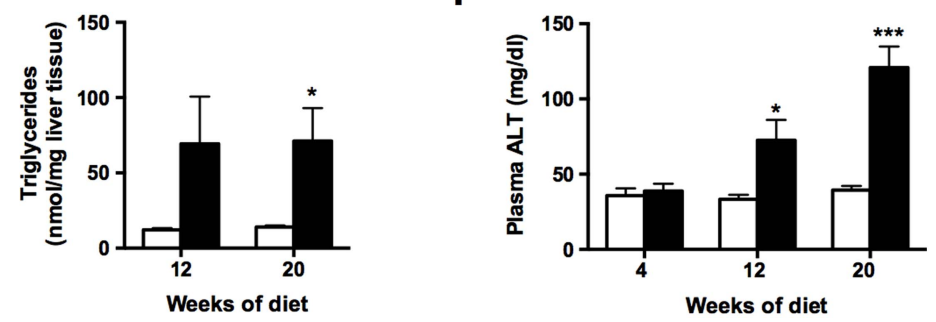

Figure 2 Liver histology in high-fat high-sucrose diet (HF-HSD) fed mice. (A) Representative H\&E images and (B) morphometric analysis of collagen proportional area of mice under HF-HSD versus chow diet at the indicated time points. (C) Collagen type I $\alpha 1$ (Col1a1), transforming growth factor $\beta 1$ (TGF- $\beta 1$ ) and tissue inhibitor of metalloproteinase 1 (Timp1) mRNA levels. (D) Liver weight. (E) Liver triglycerides. (F) Plasma alanine transaminase (ALT). Asterisks indicate significant difference versus control group at same time point. $\mathrm{N}=8$ per group per time point for all assays. Data are presented as mean. Error bars are SEM. White bars represent normal chow diet (NCD) mice; black bars represent HF-HSD mice.

RYGB in our obese NASH model induces sustained weight loss and improved insulin resistance

To explore the therapeutic capacities of RYGB on pre-existing $\mathrm{NASH}$, we performed the surgical intervention in the obese, insulin-resistant NASH model that we described in previous paragraphs. Therefore, RYGB or SHAM operation was performed in mice that in advance were fed HF-HSD for 12 weeks. After surgery, both groups received HF-HSD for 8 weeks till sacrifice (figure 5A). Survival rate in the SHAM group $(n=6)$ was $100 \%$ and $50 \%$ in the original RYGB group $(n=12)$. Causes of death were mechanical ileus $(n=3)$, small bowel ischaemia $(n=1)$ and unknown (with no clinical signs of liver dysfunction) $(n=3)$. Only RYGB mice that survived the entire experiment $(n=6)$ were included in the analysis.

Body weight at time of surgery was not different between both groups and in both groups body weight decreased in the first week after surgery due to the surgical stress. From week 2 after surgery, SHAM mice gradually regained body weight to ultimately reach their preoperative level at week 8 after surgery. RYGB mice, on the contrary, maintained their lower body weight throughout the remainder of the experiment, despite continuation of HF-HSD (figure 5B). This was reflected in a decreased fat mass in RYGB mice compared with SHAM mice, measured by DEXA 8 weeks after surgery (figure 5C). RYGB mice had a significantly better glucose tolerance compared with SHAM mice assessed by IPGTT (figure 5D). In correspondence, HOMA-IR was lower in mice that underwent RYGB versus SHAM (figure 5E). These beneficial metabolic effects of RYGB were irrespective of food intake, which was not different between both groups (figure $5 \mathrm{~F}$ ).

RYGB inhibits the progression of NASH, highlighted by an absence of fibrosis

Comparing liver histology scores of unoperated mice after 12-week HF-HSD (representing time point of surgery) with those of RYGB/SHAM mice revealed that RYGB improved steatosis and inhibited the progression of inflammation, resulting in a significantly lower NAS score in RYGB mice (figure 6A, B). We observed no differences in hepatocyte ballooning between 
A

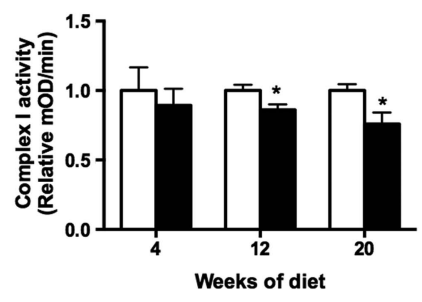

D

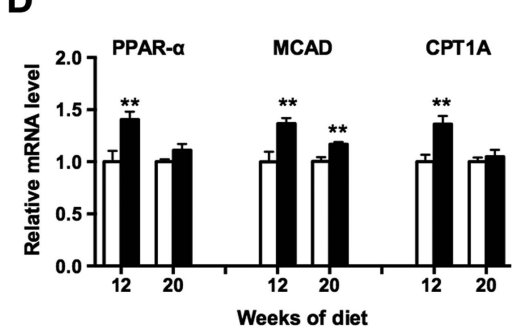

B

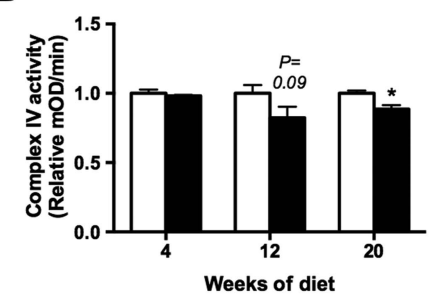

E

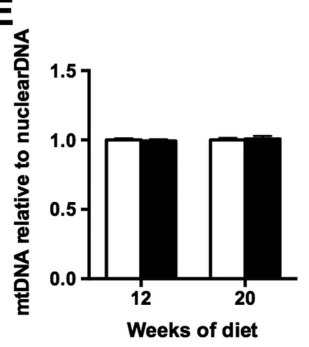

C

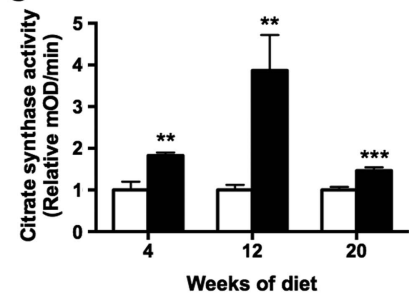

F

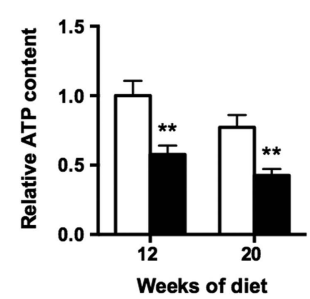

G

\section{2 weeks diet}
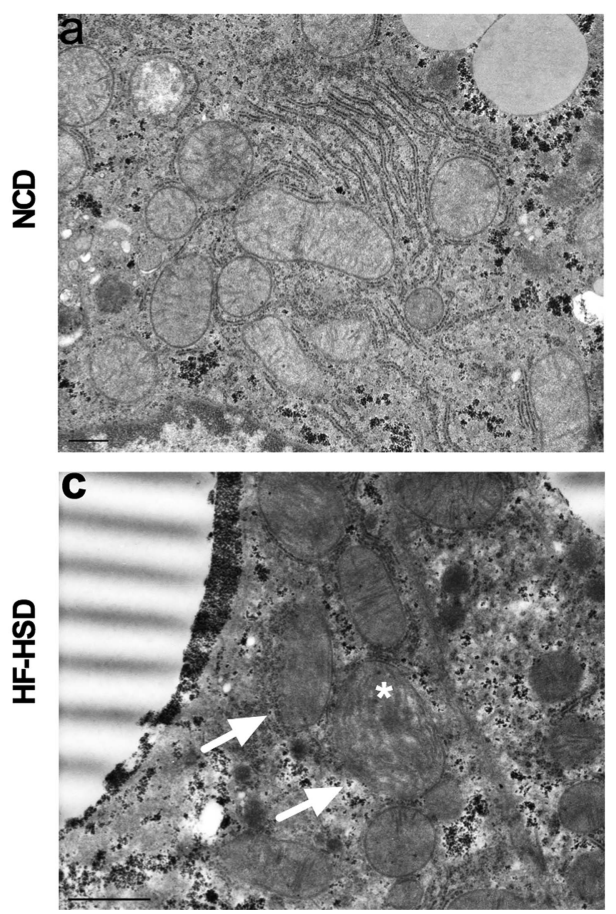

20 weeks diet
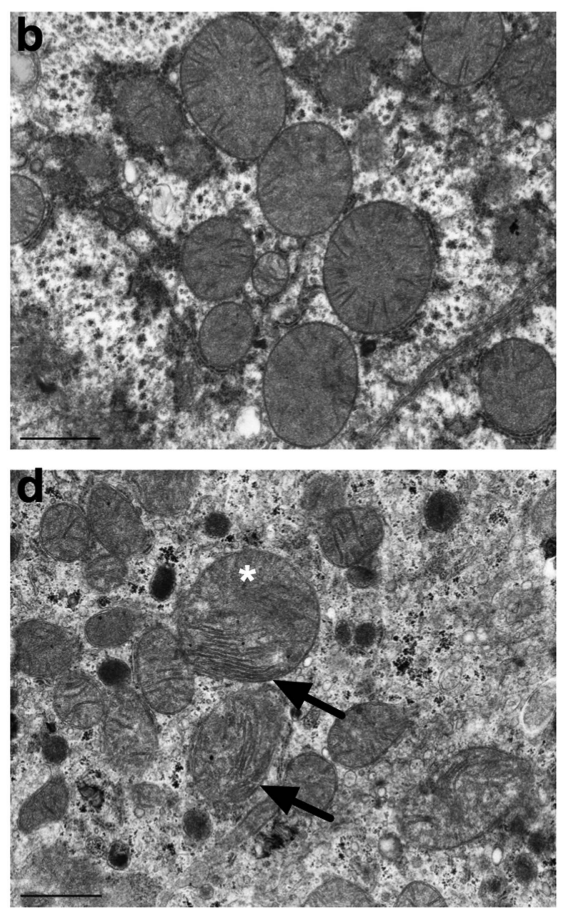

Figure 3 Hepatic mitochondrial profile of high-fat high-sucrose diet (HF-HSD) mice. (A-C) Complex I, complex IV and citrate synthase activity. Results of the control groups were normalised to 1 to correct for interassay variation. (D) Peroxisome proliferator-activated receptor $\alpha$ (PPAR- $\alpha$ ), medium chain acyl-Coenzyme A dehydrogenase (MCAD) and carnitine palmitoyltransferase 1A (CPT1A) mRNA levels. (E) Mitochondrial DNA/nuclear DNA ratio as a marker of mitochondrial content. (F) ATP content (all the samples were simultaneously measured on the same 96-well plate). (G) Representative electron micrographs $(21000 x)$ showing fine structural abnormalities of mitochondria in HF-HSD mice compared with well-preserved mitochondria in normal chow diet (NCD) mice. Swollen mitochondria with disrupted membranes (white arrows), various cristae arrangements (black arrows) and rarefied matrix (white asterisks) in HF-HSD mice are illustrated. Scale bar=1 $\mu$ m. Asterisks indicate significant difference versus control group at same time point. $\mathrm{N}=8$ per group per time point for all assays (except for electron microscopy $n=4$ per group per time point). Data are presented as mean. Error bars are SEM. White bars represent NCD mice; black bars represent HF-HSD mice.

the groups (figure 6B). Interestingly, fibrosis was absent in RYGB mice. This is in contrast to SHAM mice (five SHAM mice had fibrosis stage 2, one SHAM mouse fibrosis stage 1a) and the majority of mice that were fed HF-HSD for 12 weeks (figure 6A, C). Bit surprisingly, mRNA levels of fibrogenesis genes (Col1a1, TGF- $\beta 1$ and Timp1) were not significantly affected by RYGB (data not shown); however, semiquantitative histological scoring was confirmed by digital analysis demonstrating a significantly lower amount of collagen proportional area in mice that underwent RYGB compared with SHAM mice (figure 6D). Liver weight and hepatic triglycerides were lower in RYGB mice compared with SHAM mice, but without reaching statistical significance (see online supplementary figure S5). mRNA levels of genes involved in fatty acid uptake, lipogenesis and endoplasmic reticulum stress can be found in online supplementary figure S6. 
A

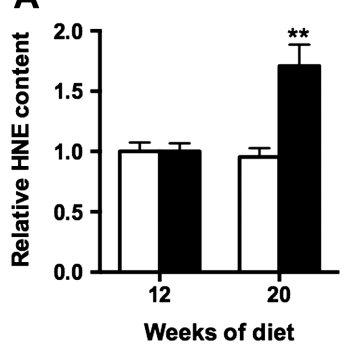

B

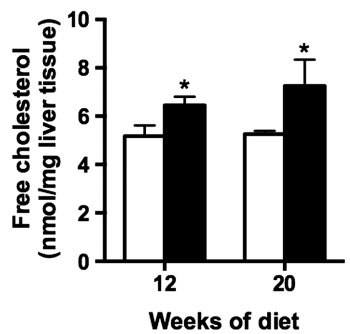

C

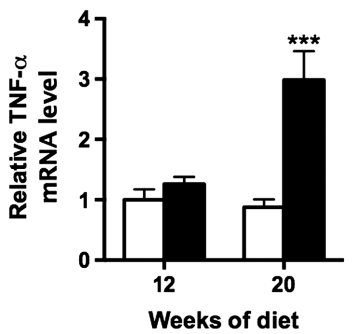

D

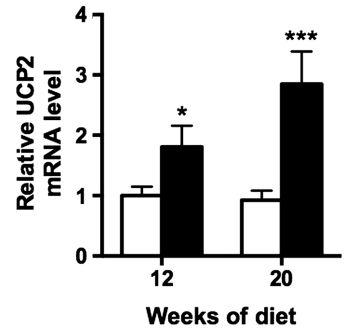

Figure 4 Hepatic modulators of mitochondrial function in high-fat high-sucrose diet (HF-HSD) mice. (A) 4-Hydroxynonenal (HNE) concentration as marker of oxidative stress. (B) Free cholesterol content. (C and D) TNF- $\alpha$ and UCP2 mRNA levels. Asterisks indicate significant difference versus control group at same time point. $\mathrm{N}=8$ per group per time point for all assays. Data are presented as mean. Error bars are SEM. White bars represent normal chow diet mice; black bars represent HF-HSD mice.

RYGB preserves hepatic MRC complex activity, restores energy output and limits the amount of oxidative stress MRC complex I activity was markedly higher in RYGB compared with SHAM mice (figure 7A). MRC complex IV activity was not significantly altered by RYGB (figure 7B). Citrate synthase activity, as well as mRNA levels of genes involved in fatty acid $\beta$-oxidation, did not differ between the surgical groups, which were both on HF-HSD during the entire experiment (figure 7C, D). Hepatic mitochondrial number (mtDNA content) was equal in both surgical groups (figure 7E). ATP content was significantly higher in RYGB mice compared with SHAM mice and mice that were fed HF-HSD for 12 weeks (representing time point surgery) (figure 7F). We observed no clear differences in mitochondrial ultrastructure between the surgical groups (data not shown). Interestingly, HNE concentration was significantly lower in RYGB mice compared with mice that underwent SHAM, indicating a limiting effect on oxidative stress by RYGB (figure $8 \mathrm{~A}$ ). In addition, TNF- $\alpha$ mRNA was significantly decreased in RYGB mice compared with SHAM mice (figure 8B), while UCP2 mRNA did not change. Finally, hepatic free cholesterol levels did not differ between RYGB and SHAM mice (see online supplementary figure S7).

\section{DISCUSSION}

In this study, we demonstrated in detail mitochondrial dysfunction in a representative NASH mouse model, reflected in a reduction of MRC complex activity and impaired energy output. RYGB prevented the progression of pre-existing NASH in these mice and in parallel beneficially influenced mitochondrial dysfunction by preserving MRC complex activity, restoring energy output and limiting the amount of oxidative stress. These data suggest that RYGB exerts a favourable effect on established NASH at least partly by modulation of hepatic mitochondrial dysfunction.

We used a mouse model closely mimicking human NASH with respect to (1) the method of induction, that is, a Western diet rich in sucrose (containing the harmful monosaccharide fructose) and saturated fatty acids; (2) the systemic metabolic profile (obesity, insulin resistance and hypercholesterolaemia); and (3) recapitulating the histological spectrum of NASH
A

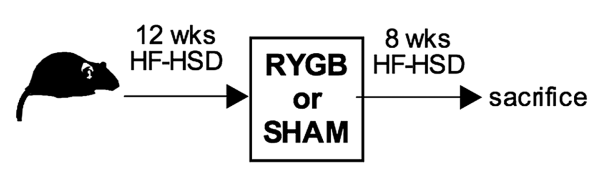

D

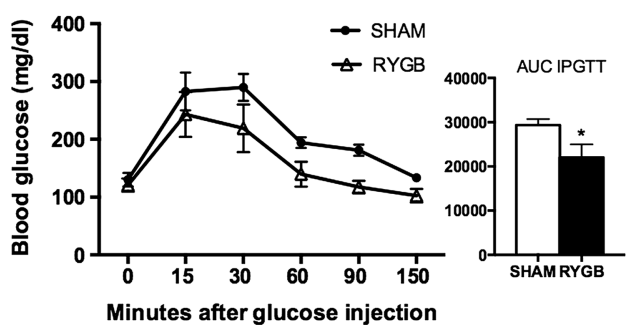

B

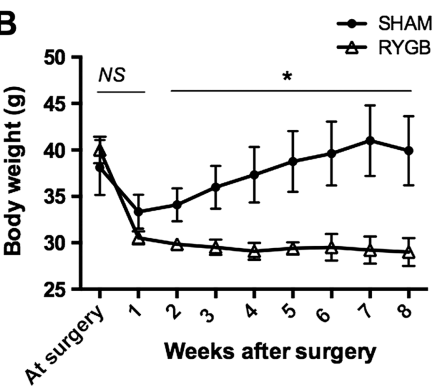

$E$

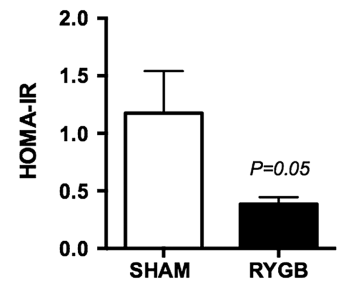

C

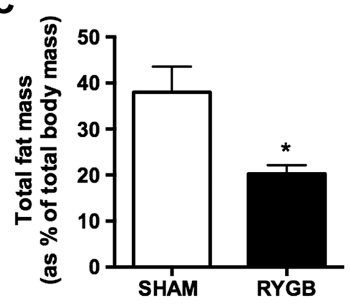

$F$

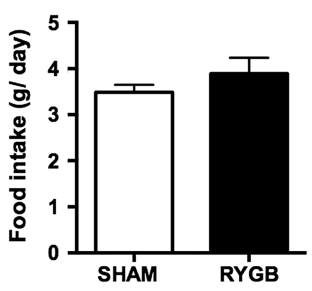

Figure 5 Systemic metabolic effects of roux-en-y gastric bypass. (A) Experimental setup. (B) Progression body weight. (C) Total fat mass measured by dual-energy X-ray absorptiometry. (D) Intraperitoneal glucose tolerance test ( $6 \mathrm{~h}$ fasting, $1 \mathrm{~g} / \mathrm{kg}$ glucose) 6 weeks after surgery. (E) Homeostasis Model of Insulin Resistance (HOMA-IR) (fasting glucose (mg/dL) xfasting insulin ( $\mathrm{ng} / \mathrm{mL}$ )/405) at sacrifice. (F) Mean food intake in postoperative period (food intake of first week after surgery was excluded from analysis). $\mathrm{N}=6$ per group for each experiment. Data are presented as mean. Error bars are SEM. 
A
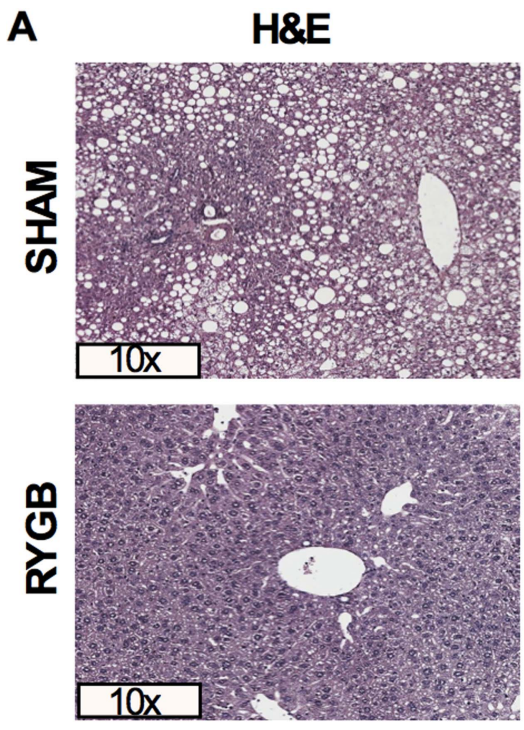

Oil Red O
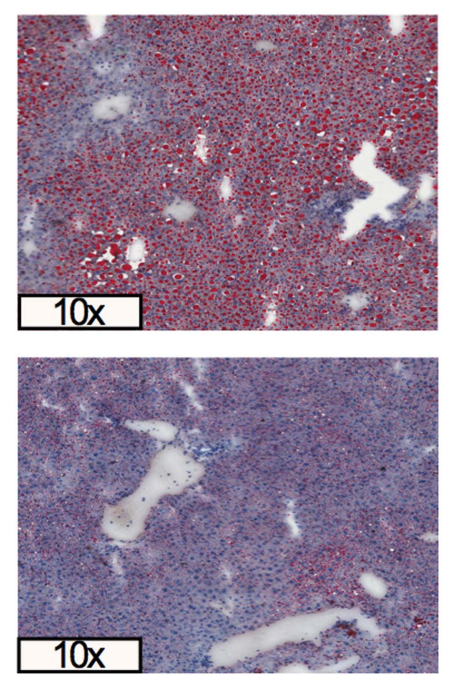

Picrosirius red
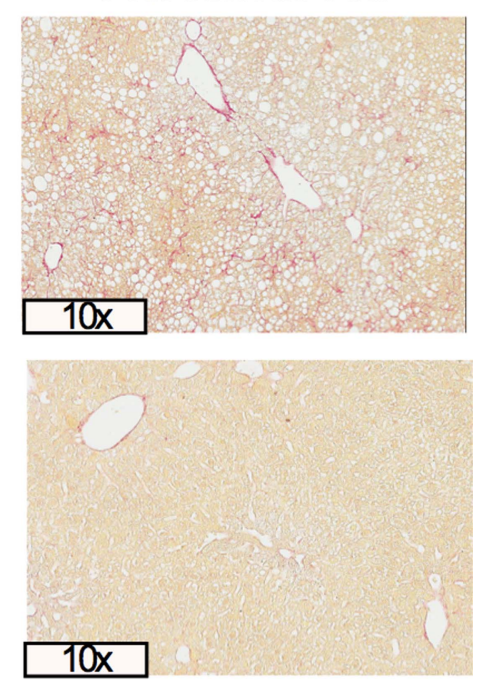
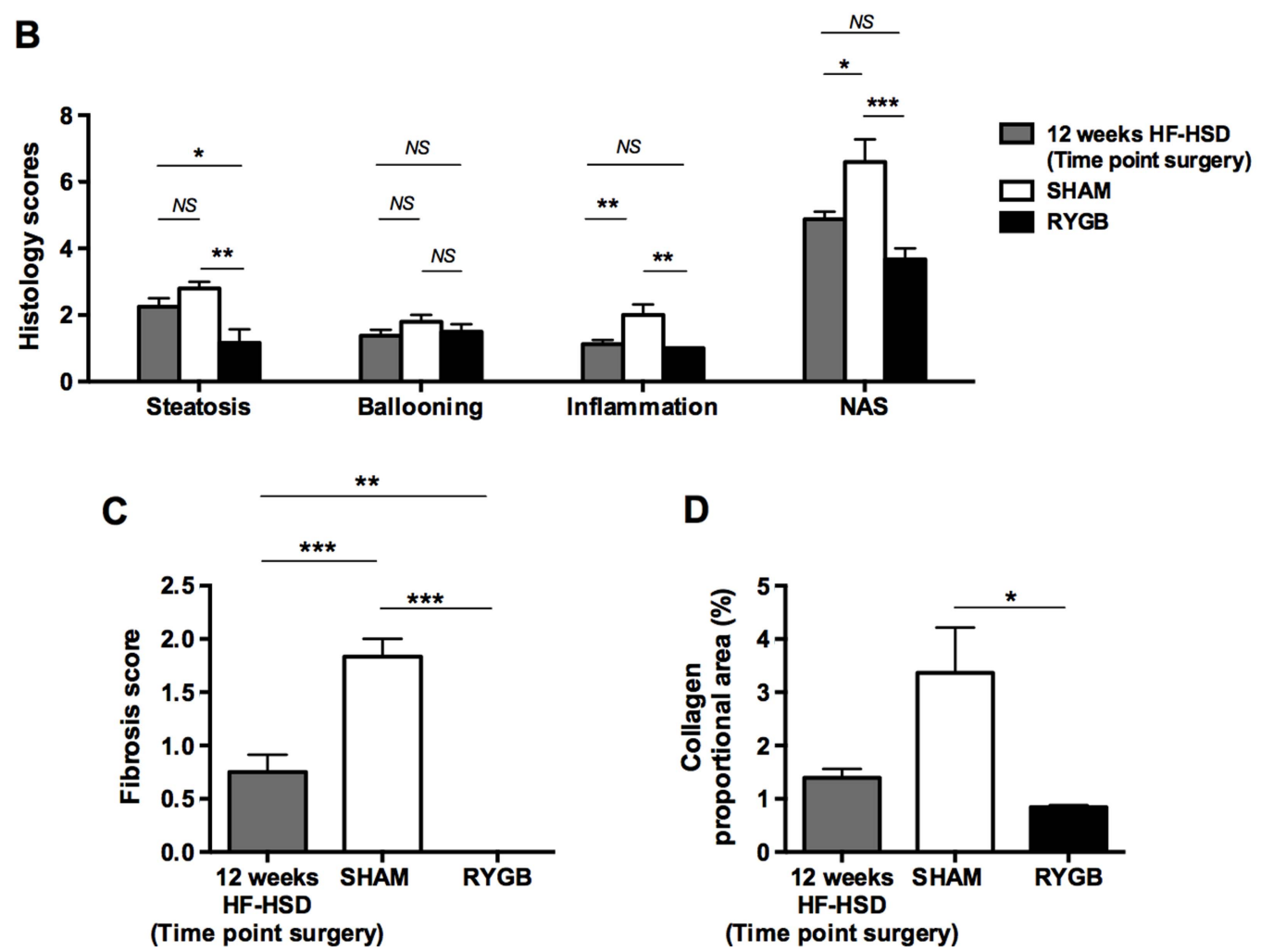

D

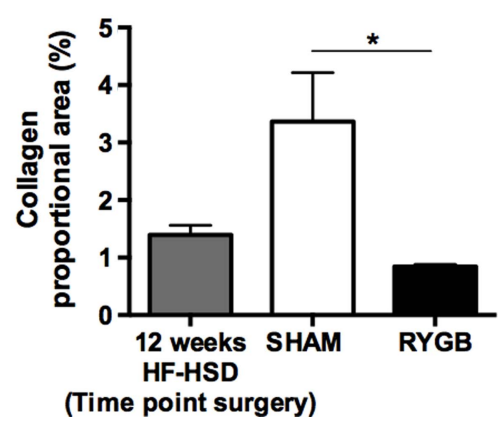

Figure 6 Histological effects of roux-en-y gastric bypass (RYGB). (A) Representative H\&E, Oil Red 0 and Picrosirius red stained images.

(B) Steatosis, hepatocyte ballooning, inflammation scores and NAS. (C) Fibrosis score. (D) Morphometric analysis of collagen proportional area. $\mathrm{N}=6$ per surgical group for each assay and $n=8$ for the 12-week high-fat high-sucrose diet (HF-HSD) group. Data are presented as mean. Error bars are SEM.

including steatosis, hepatocyte ballooning and lobular inflammation. A commercially available diet was administered to C57BL/ $6 \mathrm{~J}$ mice-a readily available strain-leading to NASH after only 12 weeks. This short feeding period in combination with the observed extent of fibrosis confers a significant benefit over three other recently described 'Western diet' mouse models of NASH. ${ }^{26-28}$ Taken together, these models facilitate clinical translation compared with other widely used NAFLD/NASH models, like methionine-deficient and choline-deficient diet mice (weight loss and absence of insulin resistance), high-fatdiet rodent models (no NASH when applying reasonable feeding periods) and leptin-deficient (ob/ob) and leptin receptor-deficient $(\mathrm{db} / \mathrm{db})$ mice (aberrant leptin signalling, no spontaneous progression to NASH and in the case of ob/ob mice even resistance to hepatic fibrosis). ${ }^{14} 15$

We observed functional as well as morphological mitochondrial changes in our HF-HSD-induced NASH model. The ATP depletion most likely stems from the reduced MRC complex activities, together with increased uncoupling of substrate oxidation from ATP synthesis, suggested by the increased UCP2 gene expression. ATP use from energy-demanding processes such as lipogenesis or gluconeogenesis, together with the fructosedriven shunt of AMP towards the production of uric acid at the expense of regeneration of ATP, can be contributory 
A

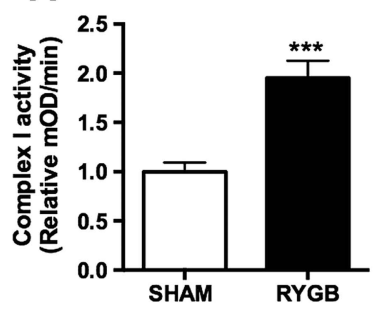

D

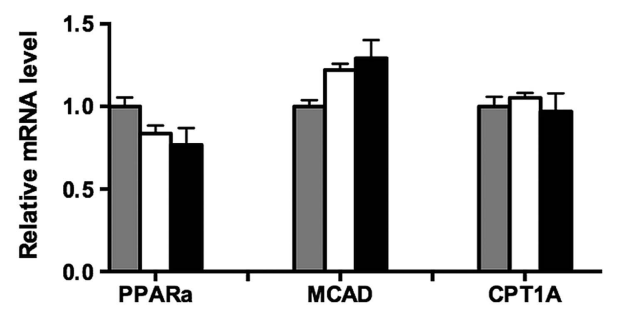

B

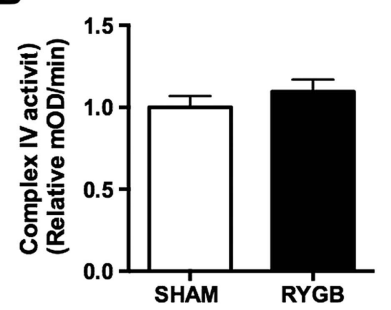

E

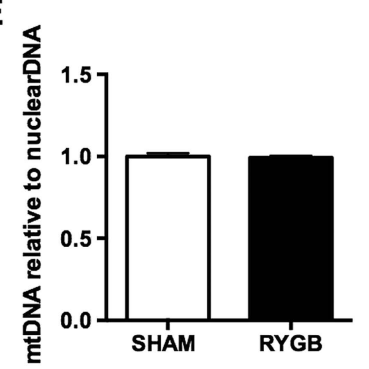

C

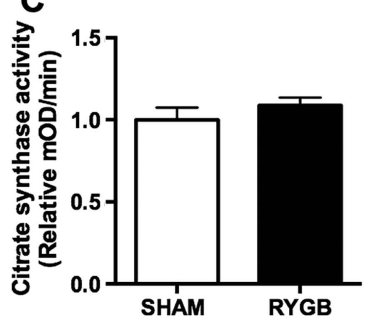

$\mathbf{F}$

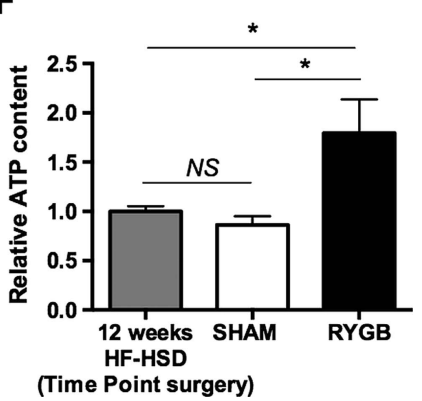

Figure 7 Hepatic mitochondrial changes after roux-en-y gastric bypass (RYGB). (A-C) Complex I, complex IV and citrate synthase activity. Results of the SHAM group were normalised to 1. (D) Peroxisome proliferator activated receptor $\alpha$ (PPAR- $\alpha$ ), medium chain acyl-Coenzyme A dehydrogenase (MCAD) and carnitine palmitoyltransferase 1A (CPT1A) mRNA levels. (E) Mitochondrial DNA/nuclear DNA ratio as a marker of mitochondrial content. (F) ATP content (all samples were simultaneously measured on the same 96 -well plate). $N=6$ per surgical group for each assay and $n=8$ for the 12-week high-fat high-sucrose diet (HF-HSD) group. Data are presented as mean. Error bars are SEM. Grey bars represent 12-week HF-HSD mice (representing time point of surgery), white bars represent SHAM mice and black bars represent RYGB mice.

explanations. $^{29}$ Our finding corresponds with the recent observation of reduced hepatic ATP concentrations in patients with obesity and type 2 diabetes with increased dietary fructose consumption. ${ }^{29}$ The differences in mitochondrial function could not be attributed to a difference in mitochondrial number between the two dietary conditions because mtDNA content was not different. In our view, citrate synthase activity, which together with fatty acid $\beta$-oxidation consistently was increased in all HF-HSD groups, represents Krebs cycle activity rather than mitochondrial number in our setup.

Subsequently, we explored the capacities of RYGB to improve the phenotype of our mouse model of NASH and acquired mitochondrial dysfunction. Numerous human trials studying the effect of bariatric surgery on NASH have been performed, but a recent Cochrane meta-analysis was unable to assess bariatric surgery as a therapeutic approach for NASH and these trials barely provided insight in molecular effects localised in the liver. $^{11} 13$ Therefore, we opted to use a mouse model that allowed in vivo and especially ex vivo experimentation under more controlled conditions. To our knowledge, this is the first time RYGB has been performed in a rodent model with established NASH. RYGB led to sustained weight loss and improved insulin sensitivity consistent with the known effects of RYGB reported in literature and observed in clinical practice, which validates our bariatric intervention. RYGB halted NASH progression and led to a striking absence of fibrosis. This is an important observation since fibrosis is the major determinant for progression to end-stage liver disease and has been put forward as the main target to combat in NASH. ${ }^{30}{ }^{31}$ Due to lack of reliable non-invasive parameters, it is difficult to determine whether the absence of fibrosis in RYGB mice represents a reversal of pre-existent fibrosis or an inhibition of progression, although the former is likely since 6/8 (75\%) mice had fibrosis after 12-week HF-HSD feeding (representing the time point of surgery).

Interestingly, RYGB attenuated mitochondrial dysfunction, which exerts a key role in NASH development and fibrogenesis. At the moment, there is no definite explanation for hepatic MRC dysfunction in NASH. ${ }^{16}$ However, some potential mediators, such as increased HNE levels, free cholesterol
Figure 8 Hepatic modulators of mitochondrial function after roux-en-y gastric bypass (RYGB).

(A) 4-Hydroxynonenal (HNE) concentration as marker of oxidative stress. (B) TNF- $\alpha$ mRNA level. $\mathrm{N}=6$ per surgical group for each assay and $n=8$ for the 12-week high-fat high-sucrose diet (HF-HSD) group. Data are presented as mean. Error bars are SEM.
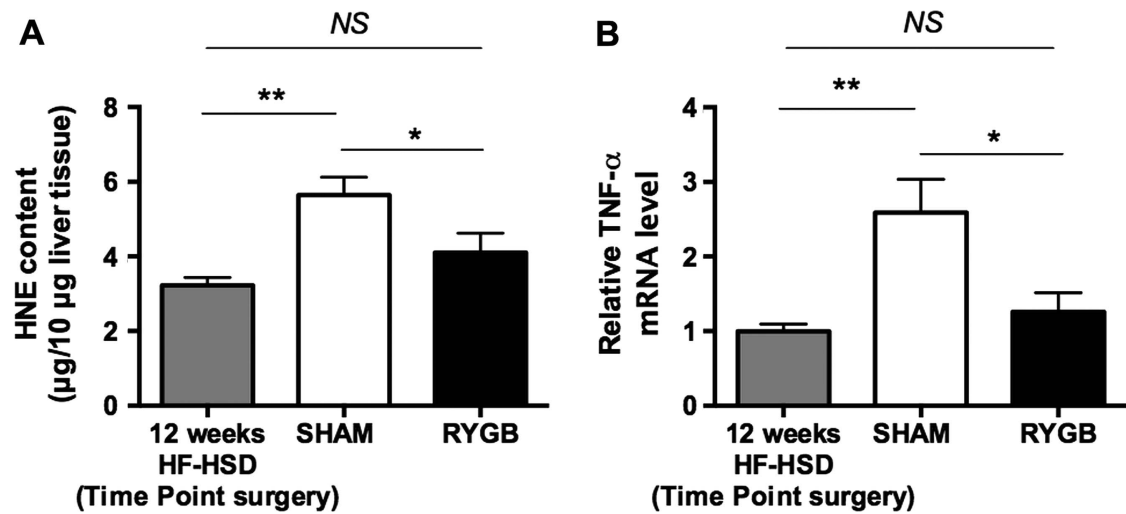
accumulation and increased TNF- $\alpha$, have been put forward, which were indeed present in our NASH model. Our results suggest that RYGB may favour MRC complex function and thereby energy output by limiting the amount of oxidative stress (lower HNE levels) and decreasing TNF- $\alpha$ gene expression. Possibly, studies in transgenic mouse models may further clarify the individual contribution of the several upstream physiological changes induced by RYGB, leading to the improvement of mitochondrial dysfunction and NASH. However, this improvement most likely is multifactorially determined. Our results expand the scarce knowledge of how bariatric surgery influences hepatic metabolic pathways. Recently, improvement of endoplasmic reticulum stress was demonstrated after bile diversion and ileal interposition (two experimental techniques mimicking the effect of bariatric surgery) in obese rats, which was accompanied by a decrease in hepatic fat. ${ }^{32}$ Bell et $a l^{34}$ showed a decrease in lipid peroxidation levels after RYGB, sleeve gastrectomy or gastric banding in humans. RYGB or sleeve gastrectomy also beneficially influenced hepatic insulin sensitivity and lipid content in patients with and without type 2 diabetes. ${ }^{35}$ Although these studies are of high importance, they were not designed to specifically evaluate the effect on (pre-existing) $\mathrm{NASH}$ and mitochondrial function.

In conclusion, our data suggest that modulation of hepatic mitochondrial dysfunction contributes to the therapeutic effect of RYBG on established NASH, in a mouse model that displays every aspect of the clinical disease. Further study on how bariatric surgery influences liver physiology on both histological and molecular level is needed, using a combination of human investigations and studies in representative animal models. This may further demarcate the position of NASH as an indication for bariatric surgery. Moreover, increased insight may facilitate the development of less invasive procedures or even pharmacological bariatric mimetics that enrich our therapeutic arsenal against the expanding clinical and public health burden of NASH.

\author{
Author affiliations \\ ${ }^{1}$ Department of Hepatology, University Hospitals KU Leuven, Leuven, Belgium \\ ${ }^{2}$ Department of Abdominal Surgery, University Hospitals KU Leuven, Leuven, \\ Belgium \\ ${ }^{3}$ Laboratory for Integrative and Systems Physiology, Ecole Polytechnique Fédérale de \\ Lausanne, Lausanne, Switzerland \\ ${ }^{4}$ Departments of Biotechnology and Molecular Medicine, A.I. Virtanen Institute for \\ Molecular Sciences, Biocenter Kuopio, University of Eastern Finland, Kuopio, Finland \\ ${ }^{5}$ Centre of Microbial and Plant Genetics (CMPG), KU Leuven, Leuven, Belgium \\ ${ }^{6}$ Department of Plant Systems Biology, Vlaams Instituut voor Biotechnologie (VIB), \\ Ghent, Belgium \\ ${ }^{7}$ Department of Pathology, Ziekenhuis Oost-Limburg, Genk, Belgium \\ ${ }^{8}$ Laboratory of Ultrastructural Neuropathology, Institute Born-Bunge (IBB), University \\ of Antwerp, Antwerp, Belgium \\ ${ }^{9}$ Laboratory of Lipid Biochemistry and Protein Interactions, KU Leuven, Leuven, \\ Belgium \\ ${ }^{10}$ Department of Pathology, Hopital Beaujon, Clichy, France \\ ${ }^{11}$ Metabolic Center, University Hospitals KU Leuven, Leuven, Belgium
}

Acknowledgements We thank E de Schryver and S Asselberghs (LIPIT) for their technical help with hepatic lipid analyses and L De Wit, K Sterck and I Bats (IBB) for their technical help with electron microscopy.

Contributors JV: design, execution of experiments, interpretation of data and writing the manuscript. ML and SF: execution of experiments and interpretation of data. EP, DR, CC-DG, PPVV, PVE and PB: execution of experiments, interpretation of data and critical revision manuscript. IvdE and PW: administrative support and execution of experiments. PS, KT, BPAC, JVP, FN and JA: interpretation of data and critical revision manuscript. DC: supervision of the project, design, interpretation of data and writing the manuscript.

Funding JV benefitted from a travel grant for a long stay abroad from the FWO Vlaanderen. EP has been funded by the Academy of Finland and Finnish Diabetes
Research Foundation. PS is supported by IWT Vlaanderen and KT by Industrial Research Fund KU Leuven (IOF-M). JA is the Nestlé Chair in Energy Metabolism and is supported by grants of the Ecole Polytechnique Fédérale de Lausanne, the ERC, the NIH and the Swiss National Science Foundation. FN and DC are fundamental clinical researchers for the FWO Vlaanderen.

Competing interests None.

Provenance and peer review Not commissioned; externally peer reviewed.

Data sharing statement Additional/supplementary data were reserved for online publication only.

\section{REFERENCES}

1 Blachier M, Leleu H, Peck-Radosavljevic M, et al. The burden of liver disease in Europe: a review of available epidemiological data. J Hepatol 2013;58:593-608.

2 Bedogni G, Miglioli L, Masutti F, et al. Prevalence of and risk factors for nonalcoholic fatty liver disease: the Dionysos nutrition and liver study. Hepatology 2005;42:44-52.

3 Cassiman D, Jaeken J. NASH may be trash. Gut 2008;57:141-4.

4 Tilg $H$, Moschen AR. Evolution of inflammation in nonalcoholic fatty liver disease: the multiple parallel hits hypothesis. Hepatology 2010;52:1836-46.

5 Charlton MR, Burns JM, Pedersen RA, et al. Frequency and outcomes of liver transplantation for nonalcoholic steatohepatitis in the United States. Gastroenterology 2011;141:1249-53.

6 Targher G, Day C, Bonora E. Risk of cardiovascular disease in patients with nonalcoholic fatty liver disease. N Engl J Med 2010;363:1341-50.

7 Centis E, Marzocchi R, Suppini A, et al. The role of lifestyle change in the prevention and treatment of NAFLD. Curr Pharm Des 2013;19:5270-9.

8 Musso G, Gambino R, Cassader M, et al. A meta-analysis of randomized trials for the treatment of nonalcoholic fatty liver disease. Hepatology 2010;52:79-104.

9 Verbeek J, Cassiman D, Lannoo M, et al. Treatment of non-alcoholic fatty liver disease: can we already face the epidemic? Acta Gastroenterol Belg 2013;76:200-9.

10 Mathurin P, Hollebecque A, Arnalsteen L, et al. Prospective study of the long-term effects of bariatric surgery on liver injury in patients without advanced disease. Gastroenterology 2009;137:532-40.

11 Lassailly G, Cailazzo R, Pattou F, et al. Bariatric surgery for curing NASH in the morbidly obese? J Hepatol 2013;58:1249-51.

12 Dixon JB, Straznicky NE, Lambert EA, et al. Surgical approaches to the treatment of obesity. Nat Rev Gastroenterol Hepatol 2011;8:429-37.

13 Chavez-Tapia N, Tellez-Avila F, Barrientos-Gutierrez T, et al. Bariatric surgery for non-alcoholic steatohepatitis in obese patients. Cochrane Database Syst Rev 2010; (1): CD007340.

14 Hebbard L, George J. Animal models of nonalcoholic fatty liver disease. Nat Rev Gastroenterol Hepatol 2011;8:35-44.

15 Anstee QM. Animal models in nonalcoholic steatohepatitis research: utility and clinical translation. Liver Int 2011;31:440-2.

16 Begriche K, Massart J, Robin MA, et al. Mitochondrial adaptations and dysfunctions in nonalcoholic fatty liver disease. Hepatology 2013;58:1497-507.

17 Andreux PA, Houtkooper RH, Auwerx J. Pharmacological approaches to restore mitochondrial function. Nat Rev Drug Discov 2013;12:465-83.

18 Matthews DR, Hosker JP, Rudenski AS, et al. Homeostasis model assessment: insulin resistance and beta-cell function from fasting plasma glucose and insulin concentrations in man. Diabetologia 1985;28:412-19.

19 Kleiner DE, Brunt EM, Van Natta M, et al. Design and validation of a histological scoring system for nonalcoholic fatty liver disease. Hepatology 2005;41:1313-21.

20 Sanyal AJ, Brunt EM, Kleiner DE, et al. Endpoints and clinical trial design for nonalcoholic steatohepatitis. Hepatology 2011;54:344-53.

21 Bedossa P, Poitou C, Veyrie N, et al. Histopathological algorithm and scoring system for evaluation of liver lesions in morbidly obese patients. Hepatology 2012;56:1751-9.

22 Bedossa $P$, Dargère $D$, Paradis V. Sampling variability of liver fibrosis in chronic hepatitis C. Hepatology 2003;38:1449-57.

23 Lagouge M, Argmann C, Gerhart-Hines Z, et al. Resveratrol improves mitochondrial function and protects against metabolic disease by activating SIRT1 and PGC-1alpha. Cell 2006;127:1109-22.

24 Gambino R, Musso G, Cassader M. Redox balance in the pathogenesis of nonalcoholic fatty liver disease: mechanisms and therapeutic opportunities. Antioxid Redox Signal 2011;15:1325-65.

25 Musso G, Gambino R, Cassader M. Cholesterol metabolism and the pathogenesis of non-alcoholic steatohepatitis. Prog Lipid Res 2013;52:175-91.

26 Charlton M, Krishnan A, Viker K, et al. Fast food diet mouse: novel small animal model of NASH with ballooning, progressive fibrosis, and high physiological fidelity to the human condition. Am J Physiol Gastrointest Liver Physiol 2011;301: G825-34.

27 Kohli R, Kirby M, Xanthakos SA, et al. High-fructose, medium chain trans fat diet induces liver fibrosis and elevates plasma coenzyme Q9 in a novel murine model of obesity and nonalcoholic steatohepatitis. Hepatology 2010;52:934-44. 
28 Ishimoto T, Lanaspa MA, Rivard CJ, et al. High fat and high sucrose (western) diet induce steatohepatitis that is dependent on fructokinase. Hepatology 2013;58:1632-43.

29 Abdelmalek MF, Lazo M, Horska A, et al. Higher dietary fructose is associated with impaired hepatic adenosine triphosphate homeostasis in obese individuals with type 2 diabetes. Hepatology 2012;56:952-60.

30 Schuppan D, Pinzani M. Anti-fibrotic therapy: lost in translation? J Hepatol 2012;56 (Suppl 1):S66-74.

31 Rosselli M, MacNaughtan J, Jalan R, et al. Beyond scoring: a modern interpretation of disease progression in chronic liver disease. Gut 2013;62: $1234-41$.
32 Kohli $R$, Setchell $K D$, Kirby $M$, et al. A surgical model in male obese rats uncovers protective effects of bile acids post-bariatric surgery. Endocrinology 2013;154:2341-51.

33 Cummings BP, Bettaieb A, Graham JL, et al. Bile-acid-mediated decrease in endoplasmic reticulum stress: a potential contributor to the metabolic benefits of ileal interposition surgery in UCD-T2DM rats. Dis Model Mech 2013;6:443-56.

34 Bell LN, Temm CJ, Saxena R, et al. Bariatric surgery-induced weight loss reduces hepatic lipid peroxidation levels and affects hepatic cytochrome P-450 protein content. Ann Surg 2010;251:1041-8.

35 Immonen $\mathrm{H}$, Hannukainen JC, lozzo $\mathrm{P}$, et al. Effect of bariatric surgery on liver glucose metabolism in morbidly obese diabetic and non-diabetic patients. $J$ Hepatol 2014;60:377-83. 


\section{GUT}

Roux-en-y gastric bypass attenuates hepatic mitochondrial dysfunction in mice with non-alcoholic steatohepatitis

Jef Verbeek, Matthias Lannoo, Eija Pirinen, Dongryeol Ryu, Pieter Spincemaille, Ingrid Vander Elst, Petra Windmolders, Karin Thevissen, Bruno P A Cammue, Jos van Pelt, Sabine Fransis, Peter Van Eyken, Chantal Ceuterick-De Groote, Paul P Van Veldhoven, Pierre Bedossa, Frederik Nevens, Johan Auwerx and David Cassiman

Gut 2015 64: 673-683 originally published online June 10, 2014 doi: 10.1136/gutjnl-2014-306748

Updated information and services can be found at:

http://gut.bmj.com/content/64/4/673

These include: Supplementary
Material

References

Email alerting service
Supplementary material can be found at: http://gut.bmj.com/content/suppl/2014/06/11/gutjnl-2014-306748.DC1. html

This article cites 34 articles, 4 of which you can access for free at: http://gut.bmj.com/content/64/4/673\#BIBL

Receive free email alerts when new articles cite this article. Sign up in the box at the top right corner of the online article.

\section{Notes}

To request permissions go to:

http://group.bmj.com/group/rights-licensing/permissions

To order reprints go to:

http://journals.bmj.com/cgi/reprintform

To subscribe to BMJ go to:

http://group.bmj.com/subscribe/ 